\title{
AN OPERATOR-THEORETIC FORMULATION OF ASYNCHRONOUS EXPONENTIAL GROWTH
}

\author{
G. F. WEBB
}

\begin{abstract}
A strongly continuous semigroup of bounded linear operators $T(t)$, $t \geqslant 0$, in the Banach space $X$ has asynchronous exponential growth with intrinsic growth constant $\lambda_{0}$ provided that there is a nonzero finite rank operator $P_{0}$ in $X$ such that $\lim _{t \rightarrow \infty} e^{-\lambda_{0} t} T(t)=P_{0}$. Necessary and sufficient conditions are established for $T(t), t \geqslant 0$, to have asynchronous exponential growth. Applications are made to a maturity-time model of cell population growth and a transition probability model of cell population growth.
\end{abstract}

1. Introduction. One of the most important phenomenon in population dynamics is the property of asynchronous or balanced exponential growth. This property is observed in many reproducing populations before the effects of crowding and resource limitation take hold. It means, roughly, that the population density function $n(x, t)$ with respect to a structure variable $x$ is asymptotic to $e^{\lambda_{0} t} n_{0}(x)$ as time $t$ becomes large. The constant $\lambda_{0}$ is intrinsic to the species in its environment. The characteristic distribution $n_{0}(x)$ depends only on the initial state. An important consequence of this phenomenon is that the proportion of the population with structure variable $x$ between two given values approaches a constant as time progresses. One important application of this phenomenon is to the so-called inverse problem $[1,4])$, that is, the use of the ultimate characteristic distribution to obtain information about various parameters in the model.

The mathematical understanding of asynchronous exponential growth began with the study of age-structured populations by F. Sharpe and A. Lotka [22]. The first rigorous proof of asynchronous exponential growth in age-structured population dynamics was given by W. Feller [6]. A general formulation of asynchronous exponential growth has been developed in the theory of branching processes $[14,15]$. Recently, it has been recognized that the idea of asynchronous exponential growth can be described in the framework of strongly continuous semigroups of bounded linear operators in Banach spaces $[3,4,7,8,9,13,17,24,25]$. The purpose of this paper is to formulate the concept of asynchronous exponential growth in this framework and to establish necessary and sufficient conditions for it to occur. We will illustrate the abstract formulation of asynchronous exponential growth in two models of proliferating cell populations. The first model is the maturity-time model

Received by the editors July 31, 1985 and, in revised form, November 14, 1986.

1980 Mathematics Subject Classification. Primary 47D05. 
of cell population growth due to S. Rubiriow [19] The second model is the trasution probability model of cell population growth due to K. Hannsgen, I. Tyson, and I. Watson [11].

2. 'The operator-theoretic formulation. We require the following standard definitions (see [24, §4.3]):

Definitron 2.1. Let $L$ be a closed linear uperator in the Baide pave $x$. If $\lambda$ is in the spectrum $\sigma(L)$, then the generalized eigenspace $\mathscr{N}_{\lambda}(L)$ is the smaliest closed subspace of $X$ containing $\bigcup_{k=1}^{\infty} N\left((\lambda I-L)^{k}\right)$. The essential speciruin $\operatorname{Eo}(L)$ is $\left\{\lambda \in \sigma(L)\right.$ : either $R(\lambda I-L)$ is not closed, $\lambda$ is a limit point of $\sigma(L)$, or $\mathscr{N}_{\lambda}(L)$ is infinite dimensional $\}$. The peripheral spectrum $\sigma_{0}(L)$ is $\left\{\lambda_{1} \in \sigma(L): \operatorname{Re} \lambda_{1}=\right.$ $\sup \{\operatorname{Re} \lambda: \lambda \in \sigma(L)\}\}$. If $L$ is bounded, then the spectral radius $r_{\sigma}(L)$ is $\sup \{|\lambda|$ : $\lambda \in \sigma(L)\}$, the essential spectral radius $r_{E \sigma}(L)$ is $\sup \{|\lambda|: \lambda \in E \sigma(L)\}$, and the measure of noncompactness is $\alpha[L]=\inf _{\varepsilon>n}\left\{L(B)\right.$ can $b_{c}$ covered by a finite number of balls of radius $\leqslant \varepsilon\}$, where $B$ is the unit ball of $X$.

The following proposition is proved in [2] (see also [24, Proposition 4.11]):

Proposition 2.1. Let $L$ be a closed linear operator in the Banach space $X$. If $\lambda_{0} \in \sigma(L)-E \sigma(L)$, then $\lambda_{0}$ is a pole of $(\lambda I-L)^{-1}$ and $\lambda_{0}$ is in the point spectrum $\operatorname{Po}(L)$.

The following proposition is proved in [24, Propositions 412,413 and 4.15$]$ :

Proposition 2.2. Let $7(t), i \geqslant 0$, be a sirongly continuous sermyroup of bounded linear operators with infinitesimal generator $A$ in the Banach space $X$.

$$
\begin{gathered}
\text { If } \gamma>\omega_{0}(A) \text {, then there exists } M_{\gamma} \geqslant 1 \text { such that }|T(t)| \leqslant M_{\gamma} e^{v:} \quad i \geqslant 0, \\
\sup _{\lambda \in \sigma(A)} \operatorname{Re} \lambda \leqslant \omega_{0}(A) \text { and } \sup _{\left.\lambda \in E \sigma_{1}, A\right)} \operatorname{Re} \lambda \leqslant \omega_{1}(A), \\
\left.r_{\sigma}(T(t))=e^{\omega_{0}(A) t} \text { and } r_{E \sigma}(T(t))=e^{\omega_{1}(A) t}, \quad t>0\right) \\
\omega_{0}(A)=\max \left\{\omega_{1}(A), \sup _{\lambda=\sigma(A)-E \sigma(A)} \operatorname{Re} \lambda\right\}
\end{gathered}
$$

If $\lambda_{0}>\gamma>\omega_{1}(A), \lambda_{0} \in \sigma(A)$, and $\sup _{\lambda \in \sigma(A)-E \sigma(A), \lambda \neq \lambda_{0}} \operatorname{Re} \lambda<$ $\gamma$, then there exists a cirect sum decomposition $X=X_{0} \oplus X_{1}$ unia associated projections $P_{1}, \quad P_{i} X=X_{i}, \quad i=0,1$, such intui $P_{0}=$ $(1 / 2 \pi i) \int_{\Gamma}(\lambda I-A)^{-1} d \lambda$ (where $\Gamma$ is a positicely criented closed curve in $\mathbf{C}$ enclosing $\lambda_{0}$ but no other point of o(A)), $P_{1}=i \cdots P_{0}$, $X_{0}=\mathscr{N}_{\lambda_{0}}(A), T(t) P_{0}=e^{t A_{1}} P_{0}, t \geqslant 0$ (where $A_{0}$ is the resiricition of A to $\left.\mathscr{N}_{\lambda_{11}}(A)\right)$, and for some constant $M_{1} \geqslant 1,\left|T(t) P_{1}\right| \leqslant M_{1} e^{\gamma t}\left|P_{1}\right|$, $t \geqslant 0$. 
If $\lambda \in P \sigma(A)$, then $e^{\lambda t} \in \operatorname{Po}(T(t)), t \geqslant 0$; if $\mu \in P \sigma(T(t))$ for some $t>0, \mu \neq 0$, then there exists $\lambda \in P \sigma(A)$ such that $e^{\lambda t}=\mu$, and $N\left(e^{\lambda t} I-T(t)\right)$ is the closed linear extension of the linearly independent subspaces $N\left(\lambda_{k} I-A\right)$, where $\lambda_{k} \in$ $P \sigma(A)$ and $e^{\lambda_{k} t}=\mu$.

DefinITION 2.2. Let $T(t), t \geqslant 0$, be a strongly continuous semigroup of bounded linear operators in the Banach space $X . T(t), t \geqslant 0$, has asynchronous exponential growth with intrinsic growth constant $\lambda_{0} \in \mathbf{R}$ provided there exists a nonzero finite rank operator $P_{0}$ in $X$ such that $\lim _{t \rightarrow \infty} e^{-\lambda_{0} t} T(t)=P_{0}$ (where the limit is in the operator norm topology).

Proposition 2.3. Let $T(t), t \geqslant 0$, be a strongly continuous semigroup of bounded linear operators with infinitesimal generator $A$ in the Banach space $X . T(t), t \geqslant 0$, has asynchronous exponential growth with intrinsic growth constant $\lambda_{0} \in \mathbf{R}$ if and only if $\omega_{1}(A)<\lambda_{0}, \sigma_{0}(A)=\left\{\lambda_{0}\right\}$, and $\lambda_{0}$ is a simple pole of $(\lambda I-A)^{-1}$.

Proof. (Necessity) Suppose that $T(t), t \geqslant 0$, has asynchronous exponential growth with intrinsic growth constant $\lambda_{0}$. It is easily seen that $P_{0}$ is a projection and $T(t) P_{0}=P_{0} T(t)=e^{\lambda_{0} t} P_{0}, t \geqslant 0$. Then, $A P_{0} x=\lim _{t \rightarrow 0}\left(T(t) P_{0} x-P_{0} x\right) / t=$ $\lambda_{0} P_{0} x, x \in X$, so that $\lambda_{0} \in P \sigma(A)$. Since $P_{0}$ is a projection, there exists a direct sum decomposition of $X$ as $P_{0} X \oplus\left(I-P_{0}\right) X$ (see [16, p. 155]). Let $\hat{X} \equiv\left(I-P_{0}\right) X$, $\hat{T}(t) \equiv e^{-\lambda_{0} t} T(t)\left(I-P_{0}\right)$, and observe that $\hat{X}$ is invariant under $\hat{T}(t)$. Consider $\hat{T}(t), t \geqslant 0$, in the Banach space $\hat{X}$ and let $\hat{A}$ be its infinitesimal generator. Observe that

$$
\lim _{t \rightarrow \infty}|\hat{T}(t)|=\lim _{t \rightarrow \infty}\left[\left|e^{-\lambda_{0} t} T(t)-P_{0}\right|+\left|\left(e^{-\lambda_{0} t} T(t)-P_{0}\right) P_{0}\right|\right]=0 .
$$

From $(2.5) e^{\omega_{0}(\hat{A}) t}=r_{\sigma}(\hat{T}(t)) \leqslant|\hat{T}(t)|$, which means $\omega_{0}(\hat{A})<0$. By (2.3) there exists $\gamma<0$ and $M_{\gamma} \geqslant 1$ such that $|\hat{T}(t)| \leqslant M_{\gamma} e^{\gamma t}, t \geqslant 0$. Since $P_{0}$ has finite rank, $T(t) P_{0}$ is compact, and so $\alpha[T(t)] \leqslant \alpha\left[T(t) P_{0}\right]+\alpha\left[T(t)\left(I-P_{0}\right)\right] \leqslant M_{\gamma} e^{\left(\lambda_{0}+\gamma\right) t}$. Thus, $\omega_{1}(A) \leqslant \lambda_{0}+\gamma<\lambda_{0}$.

Suppose that $\lambda_{1} \in \sigma(A)$ and $\operatorname{Re} \lambda_{1} \geqslant \lambda_{0}$. By (2.4) $\lambda_{1} \notin E \sigma(A)$, and by Proposition $2.1 \lambda_{1} \in \operatorname{P\sigma }(A)$. There exists $z \neq 0$ such that $T(t) z=e^{\lambda_{1} t} z$. Then,

$$
\operatorname{Re} T(t) z=e^{\operatorname{Re} \lambda_{1} t}\left[\left(\cos \operatorname{Im} \lambda_{1} t\right) \operatorname{Re} z-\left(\sin \operatorname{Im} \lambda_{1} t\right) \operatorname{Im} z\right]
$$

and

$$
\operatorname{Im} T(t) z=e^{\operatorname{Re} \lambda_{1} t}\left[\left(\cos \operatorname{Im} \lambda_{1} t\right) \operatorname{Im} z+\left(\sin \operatorname{Im} \lambda_{1} t\right) \operatorname{Re} z\right] .
$$

Since $e^{-\lambda_{0} t} \operatorname{Re} T(t) z$ and $e^{-\lambda_{0} t} \operatorname{Im} T(t) z$ converge, $\operatorname{Re} \lambda_{1}=\lambda_{0}$ and $\operatorname{Im} \lambda_{1}=0$. Thus, $\sigma_{0}(A)=\left\{\lambda_{0}\right\}$. 
Assume $\lambda_{0}$ is not a simple pole of $(\lambda I-A)^{-1}$. Since $\lambda_{0} \notin E \sigma(A)$, there exists the Laurent expansion (see [26, p. 228])

$$
\begin{aligned}
& (\lambda I-A)^{-1}=\sum_{n=-k}^{\infty}\left(\lambda-\lambda_{0}\right)^{n} A_{n}, \quad \text { where } k>1, \\
& A_{n}=(1 / 2 \pi i) \int_{\Gamma}\left(\lambda-\lambda_{0}\right)^{-n-1}(\lambda I-A)^{-1} d \lambda,
\end{aligned}
$$

$\Gamma$ is a positively oriented circle of sufficiently small radius about $\lambda_{0}$,

$$
A_{-k} \neq 0, \quad A_{-k}=\left(A-\lambda_{0} I\right)^{k-1} A_{-1}, \quad \text { and } \quad\left(A-\lambda_{0} I\right) A_{-k}=0 .
$$

Chonse $x$ such that $A_{-k} x \neq 0$ and let $y=\left(A-\lambda_{0} I\right)^{k-2} A_{-1} x$. From (2.9) $A A_{-k} x$ $=\lambda_{0} A_{-k} x$ and $A y=A_{-k} x+\lambda_{0} y$. Since

$$
\begin{aligned}
(d / d t)\left(e^{\lambda_{n} t}\left(t A_{-k} x+y\right)\right) & =\lambda_{0} e^{\lambda_{0} t}\left(t A_{-k} x+y\right)+e^{\lambda_{0} t} A_{-k} x \\
& =A\left(e^{\lambda_{0} t}\left(t A_{-k} x+y\right)\right)
\end{aligned}
$$

and the solution of the initial value problem $(d / d t) T(t) y=A T(t) y, t \geqslant 0, T(0) y$ $=y$ is unique (see [16, p. 481]), $T(t) y=e^{\lambda_{0} t}\left(t A_{-k} x+y\right)$. But $e^{-\lambda_{0} t} T(t) y$ does not converge. Thus, $\lambda_{0}$ is a simple pole.

(Sufficiency) Suppose that $\omega_{1}(A)<\lambda_{0}, \sigma_{0}(A)=\left\{\lambda_{0}\right\}$, and $\lambda_{0}$ is a simple pole of $(\lambda I-A)^{-1}$. By (2.4) and Proposition 2.1 $\lambda_{0} \in P \sigma(A)$ and $\mathscr{N}_{\lambda_{0}}(A)$ is finite dimensional. Let $\omega_{1}(A)<\gamma<\lambda_{0}$ and assume there exists an infinite sequence $\left\{\lambda_{k}\right\} \subseteq$ $\sigma(A)$ such that $\operatorname{Re} \lambda_{k} \geqslant \gamma$. Then, $\lambda_{k} \in \operatorname{Po}(A)$ and by (2.8) $e^{\lambda_{k} t} \in \operatorname{Po}(T(t))$. Fix $t>0$. If $\left\{e^{\lambda_{k} t}\right\}$ is infinite, then $\sigma(T(t))$ has an accumulation point. Thus, $r_{E \sigma}(T(t))$ $\geqslant e^{\gamma t}>e^{\omega_{1}(A) t}$, which contradicts (2.5). If $\left\{e^{\lambda_{k} t}\right\}$ is finite, then $e^{\lambda_{k} t}=\mu$ for infinitely many $k$. By (2.8) $\mathscr{N}_{\mu}(T(t))$ is infinite dimensional, since it must contain all the linearly independent manifolds $N\left(\lambda_{k} I-A\right)$ whenever $e^{\lambda_{k} t}=\mu$. Thus, $r_{E \text { o }}(T(t))$ $\geqslant \operatorname{Re} \mu \geqslant e^{\gamma t}>e^{\omega_{1}(A) t}$, which again contradicts (2.5). There must exist $\gamma>\omega_{1}(A)$ such that $\sup _{\lambda \in \sigma(A)-E \sigma(A), \lambda \neq \lambda_{0}} \operatorname{Re} \lambda<\gamma<\lambda_{0}$. There exists a direct sum decomposition of $X$ as in (2.7). Since $\lambda_{0}$ is a simple pole, $\mathscr{N}_{\lambda_{0}}(A)=N\left(\lambda_{0} I-A\right.$ ) (see [24, Proposition 4.15]) and $T(t) P_{0}=e^{\lambda_{0} t} P_{0}, t \geqslant 0$. Thus, $P_{0} \neq 0, P_{0}$ has finite rank, and

$$
\lim _{t \rightarrow \infty}\left|e^{-\lambda_{0} t} T(t)-P_{0}\right|=\lim _{t \rightarrow \infty}\left|e^{-\lambda_{0} t} T(t) P_{1}\right| \leqslant \lim _{t \rightarrow \infty} M_{1} e^{\left(\gamma-\lambda_{0}\right) t}\left|P_{1}\right|=0 .
$$

Remark 2.1. Notice from the proof of Proposition 2.3 that if $T(t), t \geqslant 0$, has asynchronous exponential growth with intrinsic growth constant $\lambda_{0}$, then (i) $P_{0}$ is the projection of $X$ onto $\mathscr{N}_{\lambda_{0}}(A)$ given in (2.7) and $T(t) P_{0}=e^{\lambda_{0} t} P_{0}, t \geqslant 0$; (ii) $\lambda_{0}$ is the dominant eigenvalue of $A$ in the sense that there exists $\delta>0$ such that $\operatorname{Re} \lambda \leqslant \lambda_{0}-\delta$ for all $\lambda \in \sigma(A), \lambda \neq \lambda_{0}$; (iii) if $\omega_{1}(A)<\gamma$ and $\lambda_{0}-\delta<\gamma<\lambda_{0}$, then there exists $M_{\gamma} \geqslant 1$ such that $\left|T(t)-e^{\lambda_{0} t} P_{0}\right| \leqslant M_{\gamma} e^{\gamma t}, t \geqslant 0$; and (iv) $P_{0}$ has rank one if and only if $\lambda_{0}$ is simple eigenvalue of $A$, that is, $\operatorname{dim} \mathscr{N}_{\lambda_{0}}(A)=1$ (see [16, p. 41]).

The following proposition provides a means to estimate $\omega_{1}(A)$ :

Proposition 2.4. Let $T(t), t \geqslant 0$, be a strongly continuous semigroup of bounded linear operators in the Banach space $X$. For sufficiently large $t$ let there exist the representation $T(t)=U(t)+V(t)$, where $|U(t)| \leqslant C e^{\gamma t}(C$ and $\gamma$ independent of $t)$ and $V(t)$ is compact. Then, $\omega_{1}(A) \leqslant \gamma$. 
Proof. From the properties of $\alpha$ ] (see [24, Proposition 4.9]) we have that $\alpha[T(t)] \leqslant \alpha[U(t)]+\alpha[V(t)]=\alpha[U(t)] \leqslant C e^{\gamma t}$ for $t$ sufficiently large. The conclusion follows immediately from (2.2).

In the case that $X$ is a Banach lattice the asymptotic behavior of strongly continuous semigroups of positive bounded linear operators has been investigated by such authors as G. Greiner [7], G. Greiner and R. Nagel [8], G. Greiner, J. Voigt, and M. Wolff [9], and W. Kerscher and R. Nagel [17]. We collect some ideas for this case into the following proposition:

Proposition 2.5. Let $T(t), t \geqslant 0$, be a strongly continuous semigroup of positive bounded linear operators with infinitesimal generator $A$ in the Banach lattice $X$. Let $\lambda_{0}=\sup \{\operatorname{Re} \lambda: \lambda \in \sigma(A)\}$ and let $\omega_{1}(A)<\lambda_{0}$. Then, (i) $\sigma_{0}(A)=\left\{\lambda_{0}\right\}$; (ii) there exists $x_{0} \in X_{+}, x_{0} \neq 0$, such that $A x_{0}=\lambda_{0} x_{0}$; (iii) if there exists a strictly positive functional $f \in X^{\prime}$ and $\lambda_{1} \in \mathbf{R}$ such that for all $x \in X_{+} \cap N\left(\lambda_{0} I-A\right)$, $\left\langle e^{-\lambda_{1} t} T(t) x, f\right\rangle$ is bounded in $t$, then $\lambda_{0} \leqslant \lambda_{1}$; (iv) if there exists a strictly positive functional $f \in X^{\prime}$ and $\lambda_{1} \in \mathbf{R}$ such that for all $x \in X_{+} \cap N\left(\lambda_{0} I-A\right), x \neq 0$, $\lim _{t \rightarrow \infty}\left\langle e^{-\lambda_{1} t} T(t) x, f\right\rangle$ exists and is positive, then $\lambda_{1}=\lambda_{0}$; and $(\mathrm{v})$ if there exists $a$ strictly positive functional $f \in X^{\prime}$ such that for all $x \in \mathscr{N}_{\lambda_{0}}(A),\left\langle e^{-\lambda_{0} t} T(t) x, f\right\rangle$ is bounded in $t$, then $\lambda_{0}$ is a simple pole of $(\lambda I-A)^{-1}$ ( $f$ is strictly positive means $\langle x, f\rangle>0$ for all $\left.x \in X_{+}, x \neq 0\right)$.

Proof. To prove (i) let $\lambda_{1} \in \sigma(A)$ such that $\operatorname{Re} \lambda_{1}=\lambda_{0}$ and $\operatorname{Im} \lambda_{1} \neq 0$. By (2.4) and Proposition 2.1 $\lambda_{1}$ is a pole of $(\lambda I-A)^{-1}$. By Theorem 1.3 in [7] $\lambda_{0}+\operatorname{im} \operatorname{Im} \lambda_{1}$ $\in \sigma_{0}(A)$ for every integer $m$. But the same argument as in the proof of Proposition 2.3 (Sufficiency) shows that $r_{E \sigma}(T(t))>e^{\omega_{1}(A) t}$, which contradicts (2.5). The proof of (ii) and (iii) uses the ideas of Greiner in [7, Corollary 1.2]. Let the Laurent expansion (2.9) hold with $k \geqslant 1$. Then, $A_{-k}=\lim _{\lambda \rightarrow \lambda_{0}^{+}}\left(\lambda-\lambda_{0}\right)^{k}(\lambda I-A)^{-1}$. In [9] it is shown that $(\lambda I-A)^{-1}$ is positive for $\lambda>\lambda_{0}$, and thus $A_{-k}$ is positive. Let $x \in X_{+}$such that $A_{-k} x \geqslant 0, A_{-k} x \neq 0$. From (2.9) $\left(A-\lambda_{0} I\right) A_{-k} x=0$, which means $T(t) A_{-k} x=e^{\lambda_{0} t} A_{-k} x$. Take $x_{0}=A_{-k} x$ to prove (ii). Since $\left\langle e^{-\lambda_{1} t} T(t) A_{-k} x, f\right\rangle=e^{\left(\lambda_{0}-\lambda_{1}\right) t}\left\langle A_{-k} x, f\right\rangle$ is bounded and $\left\langle A_{-k} x, f\right\rangle>0$, we must have $\lambda_{0} \leqslant \lambda_{1}$, which proves (iii). The proof of (iv) is similar to the proof of (iii) except that the hypothesis implies $\lim _{t \rightarrow \infty} e^{\left(\lambda_{0}-\lambda_{1}\right) t}\left\langle A_{-k} x, f\right\rangle>0$. Since $\left\langle A_{-k} x, f\right\rangle$ $>0, \lambda_{0}=\lambda_{1}$. The proof of $(\mathrm{v})$ is similar to the proof of Proposition 2.3 (Necessity). Assume that $\lambda_{0}$ is not a simple pole, so that $k>1$ in (2.9). Choose $x \in X_{+}$such that $A_{-k} x \geqslant 0, A_{-k} x \neq 0$ as in the proof of (ii) above. Let $y=\left(A-\lambda_{0} I\right)^{k-2} A_{-1} x$ and $T(t) y=e^{\lambda_{0} t}\left(t A_{-k} x+y\right)$ as in the proof of Proposition 2.3 (Necessity). Since $\left(A-\lambda_{0} I\right)^{2} y=0,\left\langle e^{-\lambda_{0} t} T(t) y, f\right\rangle=t\left\langle A_{-k} x, f\right\rangle+\langle y, f\rangle$ is bounded. Since $\left\langle A_{-k} x, f\right\rangle>0$, we obtain a contradiction.

REMARK 2.2. Propositions 2.3 and 2.5 provide sufficiency for asynchronous exponential growth of a positive semigroup in a Banach lattice: $\omega_{1}(A)<\lambda_{0} \equiv$ $\sup \{\operatorname{Re} \lambda: \lambda \in \sigma(A)\}$ and there exists a strictly positive functional $f \in X^{\prime}$ such that for $x \in \mathcal{N}_{\lambda_{0}}(A),\left\langle e^{-\lambda_{0} t} T(t) x, f\right\rangle$ is bounded in $t$. Another useful condition for this purpose is irreducibility. $T(t), t \geqslant 0$, is irreducible if for $x \in X_{+}, f \in X_{+}^{\prime}, x \neq 0$, $f \neq 0$, there exists $t>0$ such that $\langle T(t) x, f\rangle>0$. Propositions 2.3, 2.5, and 
Theorem 1.3 in [7] combine to provide another sufficient condition for asynchronous exponential growth: $\omega_{1}(A)<\lambda_{0} \equiv \sup \{\operatorname{Re} \lambda: \lambda \in \sigma(A)\}$ and $T(t), t \geqslant 0$ is irreducible. Furthermore, in this case $P_{0}$ must have rank one. We note that (i) and (ii) in Proposition 2.5 are true if $X$ is a partially ordered Banach space with ordering coming from a cone $C$ whose linear span is all of $X$ and $T(t)(C) \subset C$ for all $t \geqslant 0$; $X$ need not be a Banach lattice.

3. A maturity-time model of cell population growth. In [19] S. Rubinow presents the following model of cell population growth:

$$
\begin{gathered}
n_{t}(\tau, t)+(v(\tau) n(\tau, t))_{\tau}=-\mu(\tau) n(\tau, t), \\
v\left(\tau_{0}\right) n\left(\tau_{0}, t\right)=2 v\left(\tau_{1}\right) n\left(\tau_{1}, t\right), \\
n(\tau, 0)=\phi(\tau) .
\end{gathered}
$$

Here $n(\tau, t)$ is the density of cells at time $t$ with respect to the maturity variable $\tau \in\left[\tau_{0}, \tau_{1}\right], v(\tau)$ is the velocity of maturation, $\mu(\tau)$ is the loss rate of cells due to death or causes other than division and $\phi(\tau)$ is the initial maturity distribution of cells. Cells divide when their maturity is $\tau_{1}$. The maturity of an individual cell is connected to various physiological or biochemical changes in the cell cycle.

We assume that $v$ is continuously differentiable and positive on $\left[\tau_{0}, \tau_{1}\right]$ and $\mu$ is continuous and nonnegative on $\left[\tau_{0}, \tau_{1}\right]$. Define the Banach space $X=\{\phi \in$ $\left.C\left(\left[\tau_{0}, \tau_{1}\right]\right): v\left(\tau_{0}\right) \phi\left(\tau_{0}\right)=2 v\left(\tau_{1}\right) \phi\left(\tau_{1}\right)\right\}$ with norm $\|\phi\|=\sup _{\tau_{0} \leqslant \tau \leqslant \tau_{1}}|\phi(\tau)|$. (Notice that $X$ is not a Banach lattice with respect to the natural ordering in $C\left(\left[\tau_{0}, \tau_{1}\right]\right)$.) Let $m(\tau)=\int_{\tau_{0}}^{\tau} v(\sigma)^{-1} d \sigma, \tau_{0} \leqslant \tau \leqslant \tau_{1}(m(\tau)$ is the time required for an individual cell to mature from $\tau_{0}$ to $\tau$ ). Let

$$
\begin{aligned}
\Lambda(s, r) & =\exp \left[-\int_{r}^{s}\left(\mu(\tau)+v^{\prime}(\tau)\right) v(\tau)^{-1} d \tau\right] \\
& =\exp \left[-\int_{r}^{s} \mu(\tau) v(\tau)^{-1} d \tau\right] v(r) / v(s), \quad \tau_{0} \leqslant r \leqslant s \leqslant \tau_{1} .
\end{aligned}
$$

The solution of (3.1)--(3.3) satisfies

$$
n(\tau, t)=\left\{\begin{array}{l}
B(t-m(\tau)) \Lambda\left(\tau, \tau_{0}\right), \quad t \geqslant m(\tau), \\
\phi\left(m^{-1}(m(\tau)-t)\right) \Lambda\left(\tau, m^{-1}(m(\tau)-t)\right), \quad t \leqslant m(\tau),
\end{array}\right.
$$

where $B(t) \equiv n\left(\tau_{0}, t\right), t \geqslant 0$, satisfies

$$
B(t)=\frac{2 v\left(\tau_{1}\right)}{v\left(\tau_{0}\right)}\left\{\begin{array}{l}
B\left(t-m\left(\tau_{1}\right)\right) \Lambda\left(\tau_{1}, \tau_{0}\right), \quad t \geqslant m\left(\tau_{1}\right), \\
\phi\left(m^{-1}\left(m\left(\tau_{1}\right)-t\right)\right) \Lambda\left(\tau_{1}, m^{-1}\left(m\left(\tau_{1}\right)-t\right)\right), \quad t \leqslant m\left(\tau_{1}\right) .
\end{array}\right.
$$

The functional equation (3.5) may be solved uniquely by the method of steps for each $\phi$ in $X$. The formula (3.4) then provides a generalized solution of (3.1)-(3.3). It may be verified that these solutions form a strongly continuous semigroup of bounded linear operators $T(t), t \geqslant 0$, in $X$ by the formula $(T(t) \phi)(\tau)=n(\tau, t)$. The infinitesimal generator of $T(t), t \geqslant 0$, is $A \phi=-(v \phi)^{\prime}-\mu \phi, D(A)=\{\phi \in X$ : $\left.(v \phi)^{\prime} \in X\right\}$. 
We claim that $\sigma(A)=\operatorname{Po}(A)=\left\{\lambda_{k}: k=0, \pm 2, \pm 4, \ldots\right\}$, where $\lambda_{k} \equiv$ $\left(\ln 2-\int_{\tau_{i}}^{\tau_{1}} \mu(\tau) v(\tau)^{-1} d \tau+i k \pi\right) / m\left(\tau_{1}\right)$. To establish this claim let $\psi \in X$ and define

$$
\nrightarrow(\tau)=\Lambda\left(\tau, \tau_{0}\right) e^{-\lambda m(\tau)}\left\{\phi\left(\tau_{0}\right)+\int_{\tau_{0}}^{\tau} \Lambda(\tau, \sigma) e^{\lambda m(\sigma)} \psi(\sigma) v(\sigma)^{-1} d \sigma\right\}
$$

If $\phi \in X$, then $v\left(\tau_{n}\right) \phi\left(\tau_{\ominus}\right)=2 v\left(\tau_{1}\right) \phi\left(\tau_{1}\right)$, and from (3.6) we see that $\lambda$ must satisfy the characteristic equation

$$
2 \exp \left[-\int_{\tau_{0}}^{\tau_{1}}(\mu(\tau)+\lambda) v(\tau)^{-1} d \tau\right] \doteq 1
$$

The solutions of the characteristic equation are $\lambda=\lambda_{k}$. If $\lambda \neq \lambda_{k}$, then $\lambda \in \rho(A)$ and $(\lambda I-A) \phi=\psi$, where $\phi$ is as in (3.6) and

$$
\phi\left(\tau_{0}\right)=\frac{\left(2 v\left(\tau_{1}\right) / v\left(\tau_{0}\right)\right) \Lambda\left(\tau_{1}, \tau_{0}\right) e^{-\lambda m\left(\tau_{1}\right)} \int_{\tau_{0}}^{\tau_{1}} \Lambda\left(\tau_{1}, \sigma\right) e^{\lambda m(\sigma)} \psi(\sigma) v(\sigma)^{-1} d \sigma}{1-2 \exp \left[-\int_{\tau_{0}}^{\tau_{1}}(\mu(\tau)+\lambda) v(\tau)^{-1} d \tau\right]} .
$$

If $\lambda=\lambda_{k}$ for some even integer $k$, then $\phi(\tau)=\Lambda\left(\tau, \tau_{0}\right) \exp \left[-\lambda_{k} m(\tau)\right] \phi\left(\tau_{0}\right)$ is an eigenfunction for the eigenvalue $\lambda_{k}$ whenever $\phi\left(\tau_{0}\right) \neq 0$.

Obviously, the peripheral spectrum $\sigma_{0}(A)$ is the set of $\lambda_{k}$ above. By Proposition $2.3 T(t), t \geqslant 0$, cannot have asynchronous exponential growth. In [19] it is reasoned that the solutions of the model (3.1)-(3.3) cannot have asynchronous exponential growth, since the initial state is remembered through all successive generations. In [19] it is remarked that these solutions provide good agreement with experimental data, but that the failure to exhibit asynchronous exponential growth means this agreement persists only for a few generations.

4. A transition probability model of cell population growth. In [11] K. Hannsgen, J. Tyson, and L. Watson present a model of cell population growth with various growth laws and interdivision time probability distributions for individual cells. In [12] each cell grows linearly, so that its mass $m(t)$ at time $t$ after birth satisfies $m(t+\tau)=m(\tau)+t, t, \tau \geqslant 0$. Each cell divides into exactly two cells of equal mass after a random length of time $1+T$ comprised of a constant deterministic phase and an exponentially distributed phase with probability $\operatorname{Pr}\{T>t\}=e^{-p t}, t \geqslant 0$ ( $p=$ a positive constant). Let $n(x, t)$ have the property that $\int_{x_{1}}^{x_{2}} n(x, t) d x$ is the rate per unit time at which cells divide with mass at division between $x_{1}$ and $x_{2}$, $1 \leqslant x_{1} \leqslant x_{2}$. The function $n(x, t)$ satisfies the delay partial differential equation (4.1)

$$
n_{t}(x, t)+n_{x}(x, t)=-p n(x, t)+4 p n(2(x-1), t-1), \quad t \geqslant 0, x \geqslant 1,
$$

$$
\begin{aligned}
& n(x, t)=\phi(x, t), \quad-1 \leqslant t \leqslant 0, x \geqslant 0 \text { and } \\
& n(x, t)=0 \text { for } t>0,0 \leqslant x \leqslant 1 .
\end{aligned}
$$


In [11 and 12] the following results are established:

Proposition 4.1. Let $\phi \in C^{1}([0, \infty) \times[-1,0])$ with $\phi(0, t)=0,-1 \leqslant t \leqslant 0$, $\phi(x, 0)=0,0 \leqslant x \leqslant 1$. Then, (4.1), (4.2) has a unique solution which is also nonnegative if $\phi$ is nonnegative. Let $\lambda=\lambda_{0}$ be the unique real solution of the equation $\lambda+p=2 p e^{-\lambda}$ and let

$$
h(x)=\left\{\begin{array}{l}
N \sum_{n=0}^{\infty}(-2)^{n} c_{n} e^{-\left(\lambda_{0}+p\right) 2^{n}(x-2)}, \quad x \geqslant 2, \\
0, \quad 0 \leqslant x \leqslant 2,
\end{array}\right.
$$

where $c_{0}=1, c_{n}=1 /(2-1)(4-1) \cdots\left(2^{n}-1\right), n=1,2, \ldots$, and $N$ is a normalizing constant. Then, $h(x)>0$ for $x>2, \int_{2}^{\infty} h(x) d x=1$, and $h(x) \sim N e^{-\left(\lambda_{0}+p\right)(x-2)}$ as $x \rightarrow \infty$. If $\phi$ also satisfies $0 \leqslant \phi(x, t) \leqslant m_{0}(x)$ with $m_{0} \in C([0, \infty)) \cap L^{1}(0, \infty)$ and $m_{0}$ nonincreasing, then $e^{-\lambda_{0} t} n(\cdot, t)$ converges weakly in $L^{1}(1, \infty)$ to $c(\phi) h(\cdot)$, where

$$
c(\phi)=\frac{\int_{1}^{\infty} \phi(x, 0) d x+\left(\lambda_{0}+p\right) \int_{-1}^{0} \int_{0}^{\infty} e^{-\lambda_{0} \theta} \phi(x, \theta) d x d \theta}{1+\lambda_{0}+p} .
$$

We will use Proposition 2.3 to prove that the solutions of the model have asynchronous exponential growth with intrinsic growth constant $\lambda_{0}$ in the strong topology of an appropriately chosen Banach space. Let $0<\tau<p / 2$, let $Y=\{f \in$ $C([0, \infty)): f(0)=0$ and $\left.\lim _{x \rightarrow \infty} e^{\tau x}|f(x)|=0\right\}$ with norm $\|f\|_{Y}=\sup _{x \geqslant 0} e^{\tau x}|f(x)|$, and let $X=\{\phi \in C([-1,0] ; Y): \phi(0)(x)=0$ for $x \in[0,1]\}$ with norm $\|\phi\|_{X}=$ $\sup _{-1 \leqslant \theta \leqslant 0}\|\phi(\theta)\|_{Y}$. Define a strongly continuous semigroup of bounded linear operators in $Y$ by

$$
(S(t) f)(x)=\left\{\begin{array}{l}
e^{-p t} f(x-t), \quad x \geqslant t, \\
0, \quad 0 \leqslant x \leqslant t .
\end{array}\right.
$$

The infinitesimal generator of $S(t), t \geqslant 0$, is $B f=-f^{\prime}-p f, D(B)=\{f \in Y$ : $\left.f^{\prime} \in Y\right\}$. Define $F: X \rightarrow Y$ by

$$
(F \phi)(x)=\left\{\begin{array}{l}
4 p \phi(-1)(2(x-1)), \quad x \geqslant 1, \\
0, \quad 0 \leqslant x \leqslant 1 .
\end{array}\right.
$$

Let $\phi \in X$ and consider the problem

$$
n(t)=S(t) \phi(0)+\int_{0}^{t} S(t-s) F n_{s} d s, \quad t \geqslant 0, n_{0}=\phi,
$$

where $n:[-1, \infty) \rightarrow Y$ and $n_{t} \in X$ is defined for $t \geqslant 0$ by $n_{t}(\theta)=n(t+\theta)$, $-1 \leqslant \theta \leqslant 0$. (Notice from (4.5) and (4.6) that a solution of (4.7) satisfies $n_{t}(0)(x)=0$ for $0 \leqslant x \leqslant 1$.) By Proposition 2.1 in [23] there exists a unique solution of the problem (4.7). Define the family of operators $T(t), t \geqslant 0$, in $X$ by $T(t) \phi=n_{t}$. By Propositions 3.1 and 3.2 in [23] $T(t), t \geqslant 0$, is a strongly continuous semigroup of bounded linear operators in $X$ with infinitesimal generator $A \phi=\phi^{\prime}, D(A)=\{\phi \in$ $X: \phi^{\prime} \in X, \phi(0) \in D(B)$, and $\left.\phi^{\prime}(0)=B \phi(0)+F \phi\right\}$. By Proposition 2.3 in [23] $(T(t) \phi)(0)(x)=n(t)(0)(x) \equiv n(x, t)$ is the unique solution of (4.1), (4.2) for $\phi \in$ $D(A)$. 
Proposition 4.2. Let $\lambda=\lambda_{0}$ be the unique real solution of $\lambda+p=2 p e^{-\lambda} \cdot T(t)$, $t \geqslant 0$, has asynchronous exponential growth with intrinsic growth constant $\lambda_{0}$. Further, $\lim _{t \rightarrow \infty} e^{-\lambda_{0} t} T(t) \phi=c(\phi) \phi_{0}$ for all $\phi \in X$, where $\phi_{0}(\theta) \equiv e^{\lambda_{0} \theta} h,-1 \leqslant \theta \leqslant 0$, $c(\phi)$ is as in (4.4), and $h$ is as in (4.3).

Proof. We will apply Proposition 2.3. Since $\lambda_{0}>0$ it suffices to prove that (I) $\omega_{1}(A)<0$, (II) $\sigma_{0}(A)=\left\{\lambda_{0}\right\}$ and $\lambda_{0}$ is a simple pole of $(\lambda I-A)^{-1}$, and (III) $P_{0}$ in Definition 2.2 is given by $P_{0} \phi=c(\phi) \phi_{0}$.

(I) We will use Proposition 2.4 to show that $\omega_{1}(A) \leqslant 2 \tau-p$. Specifically, we will show that for $t \geqslant 2, T(t)=U(t)+V(t)$, where $|U(t)| \leqslant C e^{(2 \tau-p) t}$ and $V(t)$ is compact. In the calculations below it will be understood that if $f \in Y$ and $x \leqslant 0$, then $f(x)$ is taken as 0 . From (4.7) we have that for $t \geqslant 0, x \geqslant 0$

$$
\begin{aligned}
n(t)(x) & =e^{-p t} \phi(0)(x-t)+\int_{0}^{t} e^{-p(t-s)} F n_{s}(x-t+s) d s \\
& =e^{-p t} \phi(0)(x-t)+4 p \int_{0}^{t} e^{-p(t-s)} n(s-1)(2(x-t+s-1)) d s \\
& =e^{-p t} \phi(0)(x-t)+4 p \int_{-1}^{t-1} e^{-p(t-u-1)} n(u)(2(x-t+u)) d u .
\end{aligned}
$$

From (4.8) we have that for $t \geqslant 1, x \geqslant 0$

$$
\begin{array}{r}
n(t)(x)=e^{-p t} \phi(0)(x-t)+4 p \int_{-1}^{0} e^{-p(t-u-1)} \phi(u)(2(x-t+u)) d u \\
+4 p \int_{0}^{t-1} e^{-p(t-u-1)}\left\{e^{-p u} \phi(0)(2(x-t+u)-u)\right. \\
+4 p \int_{-1}^{u-1} e^{-p(u-w-1)} n(w) \\
\times(2(2(x-t+u)-u+w)) d w\} d u .
\end{array}
$$

From (4.9) we have that for $\phi \in X, t \geqslant 2,-1 \leqslant \theta \leqslant 0, x \geqslant 0$

$$
\begin{aligned}
(T(t) \phi)(\theta)(x)=n(t+\theta)(x) \\
=e^{-p(t+\theta)} \phi(0)(x-t-\theta)+4 p \int_{-1}^{0} e^{-p(t+\theta-u-1)} \phi(u)(2(x-t-\theta+u)) d u \\
\quad+4 p \int_{2(x-t-\theta)}^{2 x-t-\theta-1} e^{-p(t+\theta-1)} \phi(0)(v) d v \\
\quad+16 p^{2} \int_{2(x-t-\theta)}^{2 x-t-\theta-1} \int_{-1}^{2(t+\theta-x)+v-1} e^{-p(t+\theta-w-2)} n(w)(2(v+w)) d w d v \\
\equiv\left(U_{1}(t) \phi\right)(\theta)(x)+\left(U_{2}(t) \phi\right)(\theta)(x)+\left(U_{3}(t) \phi\right)(\theta)(x)+(V(t) \phi)(\theta)(x) .
\end{aligned}
$$

Notice that $U_{1}(t)(X) \subset X$ and

$$
\begin{aligned}
\left\|U_{1}(t) \phi\right\|_{X} & =\sup _{-1 \leqslant \theta \leqslant 0} \sup _{x \geqslant 0} e^{\tau x} e^{-p(t+\theta)}|\phi(0)(x-t-\theta)| \\
& \leqslant \sup _{-1 \leqslant \theta \leqslant 0} e^{(\tau-p)(t+\theta)}\|\phi(0)\|_{Y} \leqslant e^{(\tau-p)(t-1)}\|\phi\|_{X} .
\end{aligned}
$$


Also, $U_{2}(t)(X) \subset X$ (since for $t \geqslant 2,0 \leqslant x \leqslant \mathrm{i},-1 \leqslant u \leqslant 0$, we have that $2(x-t+u) \leqslant 0$ and $\phi(u)(2(x-t+u))=0)$. Further,

$$
\begin{aligned}
\left\|U_{2}(t)(\phi)\right\|_{X} & \leqslant \sup _{-1 \leqslant \theta \leqslant 0} \sup _{x \geqslant 0} e^{\tau x} 4 p \int_{-1}^{0} e^{p^{(t+\theta-u-1)}} e^{-2 \tau(x-t-\theta+u)}\|\phi(u)\|_{Y} d u \\
& \leqslant 4 p e^{p} e^{(2 \tau-p)(t-1)}\|\phi\|_{X} /(F-\cdots 2 \tau) .
\end{aligned}
$$

Next, $U_{3}(t)(X) \subset X$ (since for $t \geqslant 2,0 \leqslant x \leqslant 1,2(x \cdots t) \leqslant v \leqslant 2 x-1 ; 1$, we have that $v \leqslant 0$ and $\phi(0)(v)=0$ ). Further,

$$
\begin{aligned}
\left\|U_{3}(t)(\phi)\right\|_{X} & \leqslant \sup _{-1 \leqslant \theta \leqslant 0} \sup _{x \geqslant 0} e^{\tau x} 4 p \int_{2(\lambda, t-\theta)}^{2 x-i-\theta-1} e^{-p(t+\theta-1)} e^{-\tau c}\|\phi(0)\|_{Y} d v \\
& \leqslant 4 p e^{p} e^{(2 \tau-p)(t-1)}\|\phi\|_{X}^{\prime} \tau .
\end{aligned}
$$

Let $U=U_{1}+U_{2}+U_{3}$ and we have established that $|U(t)| \leqslant C e^{(2 \tau-p) t}, t \geqslant 2$, where $C$ is independent of $t$.

Observe that $V(t)(X) \subset X$, since $V(t)=T(t)-U(t)$. We must show that $V(t)$ is compact for each $t \geqslant 2$. Fix $t \geqslant 2$ and let $Q$ be a bounded subset of $X$. It suffices to show that (i) $V(t) \phi$ is uniformly bounded for $\phi \in Q$, (ii) $(V(t) \phi)(\theta)$ is equicontinuous in $\theta$ for $\phi \in Q$, (iii) $(V(t) \phi)(\theta)(x)$ is equicontinuous in $x$ in bounded intervals of $x$ for $\phi \in Q,-1 \leqslant \theta \leqslant 0$, and (iv) for $\varepsilon>0$ there exists $x_{\varepsilon}>0$ such that $e^{\tau x}|(V(t) \phi)(\theta)(x)|<\varepsilon$ for $x>x_{\mathrm{F}}$. Observe that for $\phi \in Q,-1 \leqslant \theta \leqslant 0, x \geqslant 0$, $2(x-t-\theta) \leqslant v \leqslant 2 x-t-\theta-1$, and $-1 \leqslant w \leqslant 2(t+\theta-x)+v-1$, we have $-1 \leqslant w \leqslant t-2$, so that

$$
\|n(w)\|_{Y}=\|(T(w) \phi)(0)\|_{Y} \leqslant \sup _{0 \leqslant s \leqslant t<2} \mid T(s) i\|\phi\|_{X} .
$$

Consequently, for $\phi \in Q,-1 \leqslant \theta \leqslant 0 . x \geqslant 0$

$$
\begin{aligned}
& e^{\tau x}|(V(t) \phi)(\theta)(x)| \\
& \quad \leqslant e^{\tau x} 16 p^{2} e^{-p(t+\theta-2)} \int_{2(x-t-\theta)}^{2 x-t-\theta-1} \int_{-1}^{2(t+\theta-x)+t^{t-1}} e^{p w} e^{-2 \tau(v+w)}\|n(w)\|_{Y} d w d v \\
& \quad \leqslant C e^{-3 \tau x}
\end{aligned}
$$

where $C$ is independent of $\phi, 8$, and $x$. Properties (i), (ii), (iii), and (iv) follow immediately from (4.10), (4.11), and the formula for $(V(t) \phi)(\theta)(x)$.

(II) Let $\hat{\lambda}_{0} \equiv \sup \{\operatorname{Re} \lambda: \lambda \in \sigma(A)\}$. We will use Proposition 2.5(i) and (iv) to show that $\sigma_{0}(A)=\left\{\hat{\lambda}_{0}\right\}$ and $\hat{\lambda}_{0}=\lambda_{0}$. Observe that $X$ is a Banach lattice, where $\phi_{1} \geqslant \phi_{2}$ means $\phi_{1}(\theta)(x) \geqslant \phi_{2}(\theta)(x),-1 \leqslant \theta \leqslant 0, x \geqslant 0$. Further, $T(t) \phi \geqslant 0$ for all $t \geqslant 0$ and $\phi \geqslant 0$ (which may be seen from (4.7) by using the method of steps). Suppose $\phi \in X, \phi \neq 0$, and $A \phi=\lambda \phi$. Then, $\phi(\theta)=e^{\lambda \theta} \phi(0),-1 \leqslant \theta \leqslant 0$, where $\phi(0) \in D(B)$ and $\phi^{\prime}(0)=B \phi(0)+F \phi$. Consequently,

$$
\lambda \phi(0)(x)=-\phi(0)^{\prime}(x)-p \phi(0)(x)+4 p e^{-\lambda} \phi(0)(2(x-1)), \quad x \geqslant 1,
$$

which means

$$
\phi(0)(x)=4 p e^{-\lambda} \int_{1}^{x} e^{-(\lambda+p)(x-y)} \phi(0)(2(y-1)) d y, \quad x \geqslant 1 .
$$


In [12] it is shown that a solution of (4.12) is $\phi(0)(x)=h(x)$ in (4.3) and $\lambda=\lambda_{0}$. By Proposition $4.1 h \in Y$. Then, $\lambda_{0} \in P \sigma(A)$ and $A \phi_{0}=\lambda_{0} \phi_{0}$, where $\phi_{0}(\theta)=e^{\lambda_{0} \theta} h$. Since $\omega_{1}(A)<0$ and $\lambda_{0}>0$, we have $\omega_{1}(A)<\hat{\lambda}_{0}$. By Proposition 2.5(i) $\sigma_{0}(A)=$ $\left\{\hat{\lambda}_{0}\right\}$.

Consider the scalar delay differential equation

$$
N^{\prime}(t)=-p N(t)+2 p N(t-1), \quad t \geqslant 0, \quad N_{0}=\bar{\phi} \in \bar{X} \equiv C([-1,0]) .
$$

As in [10, Chapter 7] we associate a strongly continuous semigroup of bounded linear operators $\bar{T}(t), t \geqslant 0$, in $\bar{X}$ by the formula $\bar{T}(t) \bar{\phi}=N_{t}, \bar{\phi} \in \bar{X}, t \geqslant 0$. For $\phi \in X$ define $\bar{\phi}(\theta)=\int_{0}^{\infty} \phi(\theta)(x) d x,-1 \leqslant \theta \leqslant 0$. From (4.1) we obtain that for $\phi \in D(A),(\bar{T}(t) \bar{\phi})(\theta)=\int_{0}^{\infty}(T(t) \phi)(\theta)(x) d x$. Since $D(A)$ is dense in $X$, this last formula holds for all $\phi \in X, t \geqslant 0$. The infinitesimal generator of $\bar{T}(t), t \geqslant 0$, is $\overline{A \phi}=\bar{\phi}^{\prime}, D(\bar{A})=\left\{\bar{\phi} \in \bar{X}: \bar{\phi}^{\prime} \in \bar{X}\right.$ and $\left.\bar{\phi}^{\prime}(0)=-p \bar{\phi}(0)+2 p \bar{\phi}(-1)\right\}$. Since $\bar{T}(t)$ is compact for $t \geqslant 1, \omega_{1}(\bar{A})=-\infty$. Also, $\operatorname{Po}(\bar{A})=\left\{\lambda: \lambda+p=2 p e^{-\lambda}\right\}$. For $\lambda \notin$ $\operatorname{Po}(\bar{A}), \bar{\phi} \in \bar{X}$

$$
(\lambda I-\bar{A})^{-1} \bar{\phi}(\theta)=\frac{e^{\lambda \theta}\left(\bar{\phi}(0)+2 p \int_{-1}^{0} e^{-\lambda(1+\sigma)} \bar{\phi}(\sigma) d \sigma\right)}{\lambda+p-2 p e^{-\lambda}}+\int_{\theta}^{0} e^{\lambda(\theta-\sigma) \bar{\phi}}(\sigma) d \sigma .
$$

By Proposition 2.3 and the Residue Theorem we have that for all $\bar{\phi} \in \bar{X}$

$$
\begin{gathered}
\lim _{t \rightarrow \infty}\left\|e^{-\lambda_{0} t} \bar{T}(t) \bar{\phi}-\bar{P}_{0} \bar{\phi}\right\|_{\bar{X}}=0, \\
\left(\bar{P}_{0} \bar{\phi}\right)(\theta)=\frac{e^{\lambda_{0} \theta}\left(\bar{\phi}(0)+2 p \int_{-1}^{0} e^{-\lambda_{0}(1+\sigma)} \bar{\phi}(\sigma) d \sigma\right)}{1+\lambda_{0}+p} .
\end{gathered}
$$

Define $f \in X^{\prime}$ by $\langle\phi, f\rangle \equiv \int_{-1}^{0} \int_{0}^{\infty} \phi(\theta)(x) d x d \theta, \phi \in X$, and observe that $f$ is strictly positive. From (4.14) and (4.15) we see that

$$
\lim _{t \rightarrow \infty}\left\langle e^{-\lambda_{0} t} T(t) \phi, f\right\rangle=\int_{-1}^{0}\left(\bar{P}_{0} \bar{\phi}\right)(\theta) d \theta
$$

which is positive if $\phi \geqslant 0, \phi \neq 0$. By Proposition 2.5(iv) $\lambda_{0}=\hat{\lambda}_{0}$ and by Proposition 2.5(v) $\lambda_{0}$ is a simple pole of $(\lambda I-A)^{-1}$.

(III) We will use an idea from [12, Theorem 1] to show that $N\left(\lambda_{0} I-A\right)$ is one dimensional, so that $\lambda_{0}$ is a simple eigenvalue of $A$ (see [16, p. 41]). Suppose that $A \phi=\lambda_{0} \phi$. From (4.12) we obtain

$$
\begin{aligned}
\int_{1}^{\infty}|\phi(0)(x)| d x & =4 p e^{-\lambda_{0}} \int_{1}^{\infty}\left|\int_{1}^{x} e^{-\left(\lambda_{0}+p\right)(x-y)} \phi(0)(2(y-1)) d y\right| d x \\
& \leqslant 4 p e^{-\lambda_{0}} \int_{1}^{\infty} \int_{1}^{x} e^{-\left(\lambda_{0}+p\right)(x-y)}|\phi(0)(2(y-1))| d y d x \\
& =4 p e^{-\lambda_{0}} \int_{1}^{\infty} \int_{y}^{\infty} e^{-\left(\lambda_{0}+p\right)(x-y)}|\phi(0)(2(y-1))| d x d y \\
& =\frac{4 p e^{-\lambda_{0}}}{\lambda_{0}+p} \int_{1}^{\infty}|\phi(0)(2(y-1))| d y \\
& =\int_{1}^{\infty}|\phi(0)(x)| d x .
\end{aligned}
$$


The inequality above must be an equality, so that for $x \geqslant 1$

$$
\left|\int_{1}^{x} e^{-\left(\lambda_{0}+p\right)(x-y)} \phi(0)(2(y-1)) d y\right|=\int_{1}^{x} e^{-\left(\lambda_{0}+p\right)(x-y)}|\phi(0)(2(y-1))| d y .
$$

Thus, $\phi(0)=$ const $|\phi(0)(x)|$. But the same must be true for $h-\phi(0)$. If $\int_{1}^{\infty} \phi(0)(x) d x=1$, then $\int_{1}^{\infty}(h(x)-\phi(0)(x)) d x=0$, which means $h=\phi(0)$ and $\phi_{0}=\phi$. By Proposition $2.3 \lim _{t \rightarrow \infty} e^{-\lambda_{0} t} T(t) \phi=P_{0} \phi$ for all $\phi \in X$. Let $\phi \in X$. Since $\mathscr{N}_{\lambda_{0}}(A)=N\left(\lambda_{0} I-A\right), P_{0}$ has rank one and there exists a constant $c$ such that $P_{0} \phi=c \phi_{0}$. From (4.4) and (4.16) we obtain

$$
c\left\langle\phi_{0}, f\right\rangle=\int_{-1}^{0}\left(\bar{P}_{0} \bar{\phi}\right)(\theta) d \theta=c(\phi)\left\langle\phi_{0}, f\right\rangle .
$$

Since $\phi_{0} \geqslant 0$ and $f$ is strictly positive, we must have $c=c(\phi)$.

REMARK 4.1. Notice that $T(t), t \geqslant 0$, is not irreducible, since $T(t) \phi_{0}=e^{\lambda_{0} t} \phi_{0}$, $\left(T(t) \phi_{0}\right)(0)(x)=e^{\lambda_{0} t} h(x)$, and $h(x)=0$ for $0 \leqslant x \leqslant 2$.

\section{REFERENCES}

1. G. I. Bell and E. C. Anderson, Cell growth and division. I. A mathematical model with applications to cell volume distributions in mammalian suspension cultures, Biophys. J. 7 (1967), 329-351.

2. F. E. Browder, On the spectral theory of elliptic differential operators, Math. Ann. 142 (1961), $22-130$.

3. O. Diekmann, H. Heijmans and H. Thieme, On the stability of the cell size distributions, J. Math. Biol. 19 (1984), 227-248.

4. O. Diekmann, H. Lauwerier, T. Aldenberg and J. Metz, Growth, fission, and the stable size distribution, J. Math. Biol. 18 (1983), 135-148.

5. M. Eisen, Mathematical models in cell biology, Lecture Notes in Biomathematics, Vol. 30, Springer-Verlag, Berlin, Heidelberg and New York, 1979.

6. W. Feller, On the integral equation of renewal theory, Ann. Math. Statist. 12 (1941), 243-267.

7. G. Greiner, A typical Perron-Frobenius theorem with application to an age-dependent population equation, Infinite-Dimensional Systems, Proceedings, Retzhof 1983, F. Kappel and W. Schappacher, Eds., Lecture Notes in Math., Vol. 1076, Springer-Verlag, Berlin, Heidelberg and New York, 1984.

8. G. Greiner and R. Nagel, On the stability of strongly continuous semigroups of positive operators on $L^{2}(\mu)$, Ann. Scuola Norm. Sup. Pisa Cl. Sci. (4) 10 (1983), 257-262.

9. G. Greiner, J. Voigt and M. Wolff, On the spectral bound of the generator of semigroups of positive operators, J. Operator Theory 5 (1981), 245-256.

10. J. K. Hale, Ordinary differential equations, Interscience Series on Pure and Appl. Math., Vol. 21, Wiley-Interscience, New York, 1969.

11. K. Hannsgen, J. Tyson and L. Watson, Steady-state distributions in probabilistic models of the cell division cycle, SIAM J. Appl. Math. 45 (1985), 523-540.

12. K. Hannsgen and J. Tyson, Stability of the steady-state size distribution in a model of cell growth and divison, J. Math. Biol. 22 (1985), 293-301.

13. H. Heijmans, Structured populations, linear semigroups, and positivity (to appear).

14. P. Jagers, Branching processes with biological applications, Wiley, New York, 1975.

15. B_ Balanced exponential growth: What does it mean and when is it there?, Biomathematics and Cell Kinetics, Development in Cell Biology, Vol. 2, A. Valleron and P. Macdonald, Eds., Elsevier/NorthHolland, New York and Amsterdam, 1978, 21-29.

16. T. Kato, Perturbation theory for linear operators, Springer-Verlag, Berlin, Heidelberg and New York, 1966.

17. W. Kerscher and R. Nagel, Asymptotic behavior of one-parameter semigroups of positive operators, Acta Appl. Math. 2 (1984), 297-310.

18. A. Lasota and M. C. Mackey, Globally asymptotic properties of proliferating cell populations, J. Math. Biol. 19 (1984), 43-62. 
19. S. Rubinow, Mathematical problems in the biological sciences, CBMS Regional Conf. Ser. Appl. Math., no. 10, SIAM, Philadelphia, Pa., 1973, pp. 53-73.

20. __ Age-structured equations in the theory of cell populations, Studies in Mathematical Biology, Vol. 16, Part II, Populations and Communities, S. Levin, Ed., The Mathematical Association of America, Washington, D. C., 1978, pp. 389-410.

21. H. Schaefer, Banach lattices and positive operators, Springer-Verlag, Berlin, Heidelberg and New York, 1974.

22. F. R. Sharpe and A. J. Lotka, A problem in age distributions, Philos. Mag. 21 (1911), 435-438.

23. C. C. Travis and G. F. Webb, Existence and stability for partial functional differential equation, Trans. Amer. Math. Soc. 200 (1974), 395-418.

24. G. F. Webb, Theory of nonlinear age-dependent population dynamics, Monographs and Textbooks in Pure and Appl. Math. Series, Vol. 89, Dekker, New York, 1985.

25. 269-282.

26. K. Yosida, Functional analysis, 2nd ed., Springer-Verlag, Berlin, Heidelberg and New York, 1968.

Department of Mathematics, Vanderbilt University, Nashville, Tennessee 37235 